\title{
National Policies in a Globalised World 2
}

\author{
Frances Press • Linda Mitchell
}

This is the second of two special policy editions of the International Journal of Early Childhood which canvass and analyse policy in early childhood education and care (ECEC) from around the globe. Taken together, the two editions (IJEC 45, 2, 2013; and IJEC 46,1,2014) bring contributions from Australia, Canada, Chile, Indonesia, Ireland, Malawi, New Zealand, Scotland and the United Kingdom. Utilising a range of methodologies to understand national policy contexts and analyse the consequences of policy in implementation, these collections of early childhood research enable both a sense of the flow of policy rationales and objectives across the globe as well as national specificities. In this second issue, as in the first, the reader can discern the emergence of common policy themes across national borders, and importantly, distinctiveness in the way in which seemingly common or familiar themes are expressed within national and cultural contexts. The unique policy challenges facing nation states are also brought to the fore.

This issue commences with Ali Formen and Joce Nuttall's identification and analysis of the three most prevalent discourses of early childhood policy in Indonesia: developmentalism, religious-particularly Islam-and human capital. These discourses, at times in contradiction, also converge to shape the image of children and childhood in Indonesian society in ways that are both specific to the Indonesian context and also at odds with its cultural diversity and stated commitment to religious pluralism.

Next, Helen Penn uses comparative data drawn from the OECD family database to gauge the performance of the United Kingdom's (UK) early childhood policy. In establishing the framework for her analysis, Penn highlights the potential pitfalls

\footnotetext{
F. Press $(\bowtie)$

Charles Sturt University, Bathurst, NSW, Australia

e-mail: fpress@csu.edu.au

L. Mitchell

University of Waikato, Hamilton, New Zealand
} 
and shortcomings of international comparisons: data categorisations in areas as complex as ECEC do not easily travel across national borders without transmutation of meaning. Nevertheless, through close examination of available data, Penn concludes that the UK shows a poor return on its relatively high public investment in ECEC and an unequal distribution of services. In her conclusion, she urges governments to consider increasing supply side subsidies rather than placing such heavy reliance on stimulating private investment.

Relatedly, Elizabeth Adamson and Deborah Brennan question the compatibility of social investment objectives with private investment. Designed to support productive engagement in the workforce over the long term, social investment strategies rely heavily upon the provision of high-quality early childhood education to support children's development and wellbeing over the life course as well as parental labour force participation. Drawing upon examples from the United Kingdom and Australia, Adamson and Brennan question the compatibility of social investment objectives with private investment. The policy agendas of both countries have encompassed the expansion of early childhood education and care, utilising significant public expenditure to stimulate private for-profit provision. Yet, neither country has high-quality programmes available to all who need it. Availability and quality are uneven and this unevenness threatens the achievement of stated social investment objectives.

Zsuzsa Millei and Alexandra Jones also flag the discourse of human capital in their analysis of the cosmopolitan imaginary found within the Australian Early Years Learning Framework: Belonging, Being and Becoming (EYLF) and associated education policy. They illuminate the way in which potentially alluring notions of cosmopolitanism and global citizenship (implicitly as well as explicitly referenced within such documents) are, on close examination, often tied to the interests of global capital vis-à-vis the creation of mobile, flexible and culturally 'competent' workforces, while still evoking loyalty to the unquestioned boundaries of each nation state. Utilising a radical social imaginary, they re-envisage the global spaces implied in the EYLF in ways that invoke solidarity and understanding that extends beyond national borders. They advocate for early years pedagogy to be grounded in an understanding of interconnection, interdependence and relational ethics.

The imperative to develop an understanding of the conditions facing populations outside our own nations, and to commit to social justice, is underscored by the subsequent article. Michelle Neumann, Christin McConnell and Foster Kholowa provide a powerful insight into the conditions facing children and families in Malawi, and the challenges of building a sustainable ECD system in a majority world nation. One of the world's poorest nations, with one-third of Malawi's population less than eight years of age; the health and development of large numbers of Malawian children are severely compromised. Although children's access to early child development programmes is improving, Neumann, McConnell and Kholowa provide a detailed analysis of why the quality and sustainability of many such programmes are challenged. Often housed in poor quality buildings, with little equipment, staffed by a voluntary workforce and at times unable to provide food for the children attending, many services face periodic if not permanent 
closures. The findings of Neumann and colleagues arose from 'unintended research' conducted while trying to establish a baseline data for another project. They conclude by calling for further longitudinal research based on robust baseline data, to inform the development of policy for service sustainability.

In the final article, Marcela Pardo and Christine Woodrow discuss the encroaching schoolarisation of early childhood programmes in Chile. Although Chile has a long history in the provision of early childhood education, its place on the public policy agenda has assumed greater prominence in public policy over the past two decades. This attention has been accompanied by increasing accountability measures. Further, as the nation seeks to improve educational outcomes, the monitoring of student performance within schools by external testing mandated by government, is exerting a downward pressure on early childhood education. Pardo and Woodrow examine the ways in which Chilean early childhood educators both engage with and resist the impact of such policies and the complex ways in which the policy discourse can both bolster and narrow teachers' professional identity and decision making.

Collectively, as in the previous issue, the articles reveal the influence of world policy institutions upon the shaping of early childhood education and care systems and the global flow of policy ideas and objectives. The discourse of human capital is evident in most national contexts reviewed, and as these various analyses show, has successfully generated public investment into early childhood programmes as governments around the world look to improve health and education outcomes for children. Yet, as many of the authors within this issue articulate, the parallel trend in many countries towards the privatisation of early childhood education and care seems to undermine the achievement of the objectives sought through such investment. Arguably too, the framing of early childhood policy through the rationale of maximising human capital can narrow the focus and purpose of early childhood education and care. Is the 'good childhood' or the quality of the early childhood programme only measured by the productivity of a nation and the educational attainment of children as they grow? How do early childhood educators engage with and challenge dominant discourses to generate awareness on the lived experiences of children in the present?

The diverse contributions represented within this issue provide the opportunity for readers to become familiar with significant policy questions and challenges outside their own national context. Through a preparedness to step outside our own national, cultural or paradigmatic frame, we can gain new perspectives on the local policy questions that face us. More importantly, we are encouraged to consider how early years policy and pedagogy can be developed in ways that counteract insularity and foster understanding and solidarity across cultures, religion and national borders.

\section{Frances Press and Linda Mitchell.}

Esta es la segunda de dos ediciones especiales sobre políticas, de la Revista Internacional de la Infancia Temprana, que abordan y analizan políticas en la educación para la infancia temprana y cuidados (ECEC) alrededor del mundo. Juntas, ambas ediciones (IJEC 45,2, 2013; y IJEC 46,1, 2014) nos traen contribuciones desde Australia, Canadá, Chile, Indonesia, Irlanda, Malawi, Nueva 
Zelanda, Escocia y el Reino Unido. Utilizando un rango metodológico amplio para entender los contextos políticos nacionales y analizar las consecuencias de la implementación de políticas, estas colecciones de investigación en la primera infancia permiten dar un sentido de fluir tanto a políticas racionales y objetivos alrededor del mundo como a especificidades nacionales. En esta primera edición, como en la segunda, el lector puede discernir sobre la aparición de temas de políticas comunes alrededor de fronteras internacionales y, de forma importante, con una distinción en la manera en la que temas aparentemente comunes o familiares son expresados entre contextos nacionales y culturales distintos. Los desafíos políticos únicos que enfrentan las naciones y estados son traídos a discusión.

Esta edición comienza con Ali Formen y Joce Nuttal identificando y analizando los tres discursos más prevalentes en la política para la infancia temprana en Indonesia: desarrollo de las mentalidades - fe religiosa - particularmente el Islam y el capital humano. Estos discursos, en momentos de contradicción, también convergen para adaptar la imagen de niños/as y de la infancia en la sociedad Indonesa de forma que sean específicas tanto como para el contexto Indonesio como para las posibilidades dado su diversidad cultural y declarado compromiso con el pluralismo religioso.

Luego, Helen Penn utiliza información comparativa obtenida desde la base de la OECD para afinar el actuar del Reino Unido en políticas de infancia temprana. Estableciendo el marco para su análisis, Penn destaca las potenciales trampas y deficiencias de comparaciones internacionales: la categorización de información en áreas tan complejas como ECEC, no puede recorrer fácilmente las fronteras nacionales sin transmutar su sentido. Sin embargo, luego de una examinación desde cerca de la información disponible, Penn concluye que el Reino Unido muestra un pobre retorno de su relativamente alta inversión social, hacia ECEC y una distribución desigual de servicios. En su conclusión ella impulsa a los gobiernos a considerar aumentar los recursos desde el lado de los subsidios en vez de entregar una confianza tan importante a estimular la inversión privada.

En relación con lo anterior, Elizabeth Adamson y Deborah Brennan cuestionan la compatibilidad de objetivos de inversión social con los de inversiones privadas. Diseñados para apoyar el compromiso productivo en la fuerza de trabajo a lo largo del tiempo, las estrategias de inversión social confían fuertemente en la entrega de educación para la infancia temprana de alta calidad para apoyar el desarrollo de los niños y su bienestar en el curso de sus vidas, tanto como para apoyar la fuerza de participación laboral de los padres.

Tomando los ejemplos del Reino Unido y Australia, Adamson y Brennan se cuestionaron la compatibilidad de los objetivos de inversión social con la inversión privada. Las agendas políticas de ambos países han abarcado la expansión de la educación para la infancia temprana y el cuidado, con un alto gasto público para estimular la provisión privada con fines de lucro. Sin embargo, ninguno de estos países posee programas de calidad disponibles para todos aquellos que lo necesitan. La disponibilidad y la calidad son disparejas, y esta irregularidad amenaza al logro del objetivo de inversión estatal social. 
Zsuzsa Millei and Alexandra Jones también exhiben el discurso del capital humano en su análisis del imaginario cosmopolita que se encuentra en el documento australiano Early Years Learning Framework: Belonging, Being and Becoming (EYLF) (Marco de Aprendizaje en los años tempranos: Pertenencia, Ser y Llegar a ser) y la política asociada a la educación.

Ellas iluminan la forma en que las nociones seductoras de la sociedad cosmopolita y la ciudadanía global, (explícita e implícitamente referenciadas en el documento mencionado), están, en un examen más profundo, amarradas a los intereses del capital global y a la creación de una cultura móvil, flexible y culturalmente "competente" como fuerza de trabajo útil y estrechamente ligadas a los límites y necesidades de cada nación estado.

Utilizando un imaginario social radical, se efectúa una re mirada de los espacios globales implicados en el documento EYLF en forma tal de invocar la solidaridad y la comprensión que se extiende más allá de las fronteras. Se aboga por una pedagogía de los primeros años que se sustente en la comprensión e interconexión, interdependencia y relaciones éticas.

El imperativo desarrollo y entendimiento de las condiciones que enfrentan las poblaciones fuera de nuestras naciones, y el compromiso con la justicia social son subrayados por el artículo siguiente.

Michelle Neumann, Christin McConnell y Foster Kholowa entregan una poderosa revelación de las condiciones que enfrentan niños/as y sus familias en Malawi, y los desafíos de construir un sistema Desarrollo Sustentable para la Infancia Temprana en la mayoría de las naciones del mundo. Siendo una de las naciones más pobres, donde un tercio de la población de Malawi corresponde a niños/as de menos de ocho años de edad, la salud y el desarrollo de un gran número de niños/as de Malawi se encuentran seriamente comprometidos. Aunque el acceso de los niños/as a programas de desarrollo de la infancia temprana está mejorando, Neumann, McConnell y Kholowa entregan un análisis detallado de por qué la calidad y sustentabilidad de muchos de esos programas se enfrenta a un gran desafío. Habitualmente ubicadas en edificios de muy baja calidad, con escasos recursos, cuya fuerza de trabajo está constituida por voluntarios y que, en momentos, no poseen las condiciones de proveer comida para los niños que asisten, muchos de estos servicios enfrentan cierres periódicos, si es que no se ven enfrentados a un cierre permanente. Los descubrimientos de Neumann y sus colegas se originan de una 'investigación sin intención' llevada a cabo mientras intentaban establecer una línea base de información para otro proyecto. Ellas, por consiguiente llegaron, utilizando una investigación longitudinal de mayor amplitud basada en una línea robusta de base de datos, a informar el desarrollo de políticas para sustentabilidad.

En el artículo final, Marcella Pardo y Christine Woodrow discutieron sobre la invasiva escolarización de programas para la infancia temprana en Chile. A pesar de que Chile posee una larga historia de entrega de educación para la infancia temprana, su lugar en la agenda pública ha asumido una mayor importancia en la política pública desde las últimas dos décadas. Esta medida se ha acompañado de medidas económicas crecientes. Además, a medida que la nación busca mejorar los resultados educacionales, el monitoreo de los resultados de los alumnos entre las 
escuelas por asesorías externas contratadas por el gobierno está ejerciendo una presión negativa en la educación para la infancia temprana. Pardo y Woodrow examinaron las formas en que los Educadores de Párvulos en Chile se comprometen y se resisten al impacto de tales políticas y las formas complejas en las que estos discursos políticos pueden reforzar o reducir la identidad profesional y la toma de decisiones de los Profesores/as.

En forma colectiva, como en la edición anterior, los artículos revelan la influencia de las instituciones de políticas mundiales en la formación de sistemas de educación para la infancia temprana y cuidados, y el fluir global de ideas políticas y sus objetivos. El discurso de capital humano es evidente en la mayoría de los contextos nacionales revisados, y como estos variados análisis nos muestran, han generado, exitosamente, tanto una inversión pública en programas de infancia temprana como gobiernos, alrededor del mundo, que buscan mejorar la salud y los resultados educacionales para los niños. Sin embargo, como muchos de los autores de los artículos nos muestran, la tendencia paralela en muchos países que apuntan a la privatización de la educación para la infancia temprana y el cuidado, parece socavar el logro de los objetivos que se buscan a través de tales inversiones. Podría decirse también que el marco de políticas para la infancia temprana bajo la lógica de aumentar el capital humano puede socavar el objetivo y el propósito de la educación para la infancia temprana y el cuidado. ¿Son una 'buena infancia' o la calidad de los programas de infancia temprana sólo medidos por la productividad de las naciones y por los logros educacionales de los niños a medida que crecen? ¿Cómo pueden los Educadores/as de Párvulos comprometerse con y desafiar los discursos dominantes para generar conciencia de las experiencias vividas por los niños/as en el presente?

Las diversas contribuciones representadas a lo largo de esta edición proveen la oportunidad a los lectores de familiarizarse con interrogantes políticas significativas y desafíos fuera de su propio contexto. A través de una adecuada preparación para dar un paso fuera de nuestro propio contexto nacional, cultural o paradigmático, podemos adquirir nuevas perspectivas de las interrogantes políticas locales que enfrentamos. Más importante aún, nos vemos animados a considerar cómo la pedagogía y las políticas para los primeros años pueden desarrollarse de formas en que contrarresten la falta de criterio y fomenten el entendimiento y la solidaridad entre culturas, religiones y fronteras.

Frances Press and Linda Mitchell.

Ce numéro spécial de la Revue internationale de l'enfance préscolaire est le deuxième qui esquisse et analyse les politiques en éducation et accueil de la jeune enfance (EAJE) autour du monde. Mis ensemble, les deux numéros spéciaux (vol. 45, no.2, 2013 et vol.46 no.1, 2014) présentent des contributions d'Australie, du Canada, du Chili, de l'Indonésie, de l'Irlande, du Malawi, de Nouvelle-Zélande, d'Écosse et du Royaume Uni. Au moyen d'un spectre de méthodologies visant à comprendre les contextes des politiques nationales et à analyser les conséquences de leur mise en place, ces collections de recherches préscolaires permettent de saisir le sens des argumentaires et des objectifs des politiques autour du monde de même que leurs particularités nationales. Dans ce numéro comme dans le précédent, le lecteur peut voir émerger des thèmes communs aux diverses politiques nationales, mais 
surtout une façon différente dont des thèmes apparemment communs ou familiers s'expriment dans les contextes culturels nationaux. Les défis uniques auxquels les états nations font face sont aussi mis de l'avant.

Ce numéro commence par la description et l'analyse de trois des discours les plus prévalents dans les politiques préscolaires en Indonésie : le développementalisme, la foi religieuse - particulièrement l'Islam- et le capital humain, par Ali Formen et Joce Nuttal. Ces discours, parfois contradictoires, convergent aussi pour façonner une image des enfants et de l'enfance dans la société indonésienne de manière à la fois spécifique au contexte indonésien mais aussi en porte à faux de sa diversité culturelle et de l'affirmation de son engagement envers le pluralisme religieux.

Ensuite, Helen Penn utilise des données comparatives, tirées de la base de données sur les familles de l'OCDE, pour jauger la performance de la politique de la jeune enfance du Royaume Uni. En posant le cadre de son analyse, Penn souligne les pièges et les lacunes des comparaisons internationales: les catégories de données dans des domaines aussi complexes que l'EAJE ne traversent pas facilement les frontières nationales sans transmutation de sens. Par un examen serré des données disponibles, Penn conclut néanmoins que le Royaume Uni démontre un faible retour sur son investissement relativement élevé en EAJE ainsi qu'une distribution inéquitable de services. Dans sa conclusion, elle incite les gouvernements à penser à augmenter les subventions aux équipements additionnels plutôt qu'à faire autant confiance à la stimulation de l'investissement privé.

Dans le même ordre d'idée, Elizabeth Adamson et Deborah Brennan s'interrogent sur la compatibilité d'objectifs de l'investissement social avec l'investissement privé. Conçues pour soutenir à long terme l'engagement productif dans la main d'œuvre, les stratégies d'investissement social s'appuient largement sur l'offre de services d'éducation préscolaire de grande qualité afin de favoriser le développement et le bien-être des enfants tout au long de leur vie ainsi que la participation des parents au marché du travail. À partir d'exemples provenant du Royaume Uni et de l'Australie, Adamson et Brennan mettent en question la compatibilité d'objectifs d'investissement social avec l'investissement privé. Les politiques des deux pays englobent l'expansion de l'éducation et de l'accueil des jeunes enfants, au moyen de dépenses publiques considérables pour stimuler l'offre de services à but lucratif privés. Pourtant aucun des pays n'a de services de qualité accessibles à tous ceux qui en ont besoin. L'accessibilité et la qualité sont inégales et cette inégalité menace l'atteinte des objectifs d'investissement social déclarés.

Zsuzsa Millei et Alexandra Jones signalent aussi le discours sur le capital humain dans leur analyse de l'imaginaire cosmopolite trouvé dans l'Early Years Learning Framework: Belonging, Being and Becoming (EYLF) australien et la politique éducative associée. Elles éclairent la façon dont les notions potentiellement séduisantes de cosmopolitisme et de citoyenneté du monde (auxquelles ces documents font implicitement et explicitement référence) sont, après examen serré, souvent liées aux intérêts du capital mondial envers la création d'une main d'œuvre mobile, flexible et culturellement «compétente », tout en évoquant une loyauté envers les frontières sans équivoque des états nations.

La nécessité de développer une compréhension des conditions auxquelles les populations font face hors de nos propres pays et de nous engager envers la justice 
sociale est soulignée dans l'article suivant. Michelle Neumann, Christin McConnell et Foster Kholowa donnent un puissant éclairage sur les conditions vécues par les enfants et les familles du Malawi et sur les défis de l'élaboration d'un système préscolaire durable dans un pays du monde majoritaire. Dans l'un des pays les plus pauvres du monde, un tiers de la population du Malawi ayant moins de huit ans, la santé et le développement d'un grand nombre d'enfants sont gravement compromis. Même si l'accès des enfants à des services de développement préscolaire s'améliore, Neumann, McConnell et Kholowa présentent une analyse détaillée des raisons pour lesquelles la qualité et la pérennité de plusieurs de ces services sont mises à l'épreuve. Souvent logés dans des bâtiments de faible qualité, peu équipés, dotés d'une main d'œuvre bénévole parfois incapable de nourrir les enfants qui les fréquentent, plusieurs services font face à des fermetures temporaires si non permanentes. Les résultats de Neumann et ses collègues ont émergé d'une recherche «non intentionnelle » menée alors qu'ils cherchaient à établir des données de base pour un autre projet. Ils concluent en lançant un appel à recherche longitudinale basée sur des données de base solides pour documenter l'élaboration de politiques visant la pérennité des services.

Dans le dernier article, Marcella Pardo et Christine Woodrow discutent de la scolarisation qui empiète sur les programmes préscolaires au Chili. Même si le Chili a une longue histoire d'offre de services d'éducation des jeunes enfants, la place de ces derniers à l'agenda des politiques publiques est devenue plus proéminente au cours des deux dernières décennies. Cette attention a été accompagnée de mesures croissantes de rendement. De plus, comme le pays cherche à améliorer les résultats scolaires, la supervision dans les écoles de la performance des élèves par des tests externes exigés par le gouvernement exerce une pression qui s'exerce jusqu'à l'éducation des jeunes enfants. Pardo et Woodrow examinent la façon dont les éducatrices et éducateurs chiliens à la fois s'engagent et résistent à l'impact de telles politiques ainsi que la manière complexe dont le discours politique peut tant renforcer que réduire l'identité professionnelle et la prise de décision des enseignantes et enseignants.

Collectivement, comme dans le numéro précédent, les articles révèlent l'influence des institutions politiques mondiales sur le façonnement des systèmes d'éducation et d'accueil des jeunes enfants et le mouvement des idées et des objectifs politiques mondiaux. Le discours du capital humain est évident dans la plupart des contextes nationaux recensés et, comme l'indiquent ces diverses analyses, et a généré avec succès l'investissement dans les services préscolaires alors que les gouvernements du monde envisagent d'améliorer la santé et l'éducation des enfants. Pourtant, comme plusieurs auteurs le signalent dans ce numéro, la tendance parallèle de plusieurs pays à la privatisation de l'éducation et l'accueil des jeunes enfants semble saper l'atteinte des objectifs visés par ce même investissement. Aussi discutable, l'encadrement des politiques de la jeune enfance par l'argumentaire de la maximisation du capital humain peut réduire la focalisation et le but de l'éducation et l'accueil des jeunes enfants. La «bonne enfance » ou la qualité du programme préscolaire est-elle mesurée uniquement par la productivité d'un pays et le niveau de scolarité atteint par les enfants lorsqu'ils grandissent? Comment les éducateurs et éducatrices de jeunes enfants s'engagent-ils dans les 
discours dominants ou les défient-ils pour faire connaitre les expériences vécues présentement par les enfants?

Les diverses contributions représentées dans ce numéro donnent aux lecteurs l'occasion de se familiariser avec d'importants problèmes et questions politiques hors de leur propre situation nationale. Lorsque nous sommes prêts à nous déplacer hors de notre propre cadre national, culturel ou paradigmatique, nous pouvons avoir de nouvelles perspectives sur les questions politiques locales auxquelles nous sommes confrontés. Mais surtout, nous sommes encouragés à envisager comment la politique et la pédagogie peuvent être élaborées de façon à contrer l'isolement et favoriser la compréhension et la solidarité à travers les frontières nationales, des cultures et des religions.

Frances Press and Linda Mitchell. 